\title{
Effects of vessels on behaviour patterns of individual southern resident killer whales Orcinus orca
}

\author{
Rob Williams ${ }^{1,5, *, * *}$, David E. Bain ${ }^{2, * *}$, Jodi C. Smith ${ }^{3}$, David Lusseau ${ }^{4}$ \\ ${ }^{1}$ Sea Mammal Research Unit, Gatty Marine Laboratory, St. Andrews University, St Andrews, Fife KY16 8LB, UK \\ ${ }^{2}$ University of Washington, Friday Harbor Laboratories, 620 University Road, Friday Harbor, Washington 98250, USA \\ ${ }^{3}$ Coastal-Marine Research Group, Institute of Natural Resources, Massey University at Albany, Private Bag 102904 , \\ North Shore MSC, New Zealand \\ ${ }^{4}$ University of Aberdeen, Institute of Biological and Environmental Sciences, Tillydrone Avenue, Aberdeen AB24 2TZ, UK \\ ${ }^{5}$ Present address: Marine Mammal Research Unit, University of British Columbia, 2202 Main Mall, Vancouver, \\ British Columbia V6T 1Z4, Canada
}

\begin{abstract}
Southern resident killer whales numbered only 84 ind. in 2004. Disturbance by vessels may be a factor in the population's endangered status. To determine the importance of this factor, we compared behaviour in the presence and absence of vessels from 2003 to 2005 at 2 different sites along San Juan Island, Washington State, USA. Theodolite tracks were summarised in terms of swimming path directness and deviation indices, travel speed, and rates of respiration and surface active display behaviours. Vessel number and proximity were used in a generalised additive modelling framework as candidate explanatory variables for differences in whale behaviour, along with natural factors. Path directness varied with number of vessels and proximity to vessels. The increased distance that whales travelled in the presence of vessels could have resulted in increased energy expenditure relative to whales that could rest while waiting for affected whales to catch up. The likelihood and rate of surface active behaviour varied with number of vessels. Number and proximity of vessels were also related to variability in respiratory intervals, path deviation index and swimming speed. The high proportion of time that southern resident killer whales spend during summer in proximity to vessels raises the possibility that the short-term behavioural changes reported here may have biologically significant consequences.
\end{abstract}

KEY WORDS: Whale watching $\cdot$ Killer whale $\cdot$ Disturbance $\cdot$ Behavioural disruption $\cdot$ Ecotourism

\section{INTRODUCTION}

The eastern North Pacific 'southern resident' stock of killer whales Orcinus orca declined to a low of 79 individuals in 2001 (van Ginneken et al. 2002). The population experienced a $20 \%$ decline between 1996 and 2001, which resulted in their listing as 'depleted' under the US Marine Mammal Protection Act, and 'Endangered' under the US and Washington State Endangered Species Acts and Canada's Species at Risk Act (Krahn et al. 2004, Wiles 2004, US Fish and Wildlife Service 2005, Fisheries and Oceans Canada 2008). The causes of this decline are uncertain, but candidate explanations include reduction in prey resources, presence of toxic chemicals and disturbance from vessel traffic. Vessel traffic may have contributed to the decline through a variety of mechanisms, including collisions, toxins from unburned fuel and exhaust, stress, and reduced foraging efficiency due to masking of echolocation signals. Short-term behavioural responses by whales to boats may result in increased energy expenditure, or disrupt feeding activity, which may reduce energy acquisition (Williams et al. 2006, Lusseau et al. 2009). Energetic mechanisms for impact 
are of particular concern because southern resident killer whales may be food-limited (Fisheries and Oceans Canada 2008).

Behavioural responses of cetaceans to vessel traffic vary within and between species, and include changes in respiration patterns, surface active behaviours, swimming velocity, vocal behaviour, activity state, inter-individual spacing, wake riding, approach and avoidance, and displacement from habitat. Studies on killer whales have shown that boats can elicit a number of short-term behavioural responses. Experiments conducted on the adjacent northern resident killer whale population showed that whales adopted less predictable paths in the presence of vessels than they were following before the boat arrived (Williams et al. 2002a,b). For southern resident killer whales, even subtle behavioural responses to boats have not been reported in the primary literature. This is a critical area of study because the San Juan and Gulf Island waters have high levels of vessel traffic and these whales are in the presence of vessels (including those not focused on whale watching) during much of the day. In terms of the candidate explanations for the decline, vessel traffic is a priority research topic because it is the factor that lends itself most readily to management and mitigation. This study addresses relationships between vessel activity and southern resident killer whale behaviour.

\section{MATERIALS AND METHODS}

Field methods. From 28 July to 30 September 2003, 1 May to 31 August 2004, and 15 May to 31 July 2005, a land-based team of observers monitored behaviour of whales and activity of boats from 2 study sites (Fig. 1). The 'North Site' was located at $48^{\circ} 30.561^{\prime} \mathrm{N}$, $123^{\circ} 8.494^{\prime} \mathrm{W}$ at an altitude of approximately $99 \mathrm{~m}$ above mean lower low water. The 'South Site' was located at Mt. Finlayson $\left(48^{\circ} 27.421^{\prime} \mathrm{N}, 122^{\circ} 59.401^{\prime} \mathrm{W}\right)$, near the southeast tip of San Juan Island. The South Site was located at a height of $72 \mathrm{~m}$ and the view of the eastern portion of Juan de Fuca Strait was unobstructed. The theodolite height was determined using the method of Williams et al. $(2002 a, b)$. We chose the combination of these 2 sites so as maximize sample size. For the 3 study periods combined, data were obtained on 128 d over approximately 9 mo in the field.

Theodolite tracking of focal individuals and boats. Two land-based teams of observers were established between the 2 study sites to maximise sample size. The teams recorded boat and whale positions and activity using a Pentax ETH-10D theodolite interfaced to a personal computer running Theoprog (Williams et al. 2002a,b, Williams \& Ashe 2007), a Bushnell 40× spotting scope, binoculars, and a mini-DV camera (DeNardo et al. 2001).

As whales entered the field of view from a study site, a focal individual was selected and indentified by comparing natural markings identified to a published photo-identification catalogue compiled by the Center for Whale Research (e.g. van Ginneken et al. 2002, with annual updates). Whales were tracked for at least $800 \mathrm{~s}$ because short observation sessions have been shown to yield biased estimates of respiration rate (Kriete 1995). After a tracking session was completed, a new focal individual was selected, when possible. Individuals were selected haphazardly, but were drawn as evenly as practicable from all pods, age, and sex classes. Observers chose individuals that would not be confused with other individuals nearby and that were sufficiently close to shore to be accurately identified (typically within $3 \mathrm{~km}$ ). The theodolite was used to record position of the focal individual at the time of every surfacing. The spotting scope and computer operators, who had a wider field of view, watched for surfacings missed by the theodolite operator, to ensure an accurate record of respiration rate and surface active behaviour. While the focal whale appeared to be on a long dive, the theodolite operator recorded vessel positions. During periods of intense vessel activity, a second theodolite team tracked only vessels, so that the primary theodolite operator could focus solely on measuring whale behaviour (the top priority). While the theodolite operator recorded the position of boats, the spotting scope operator scanned for the whale; the probability of missing a surfacing was expected to be low overall, but did not vary with traffic level.

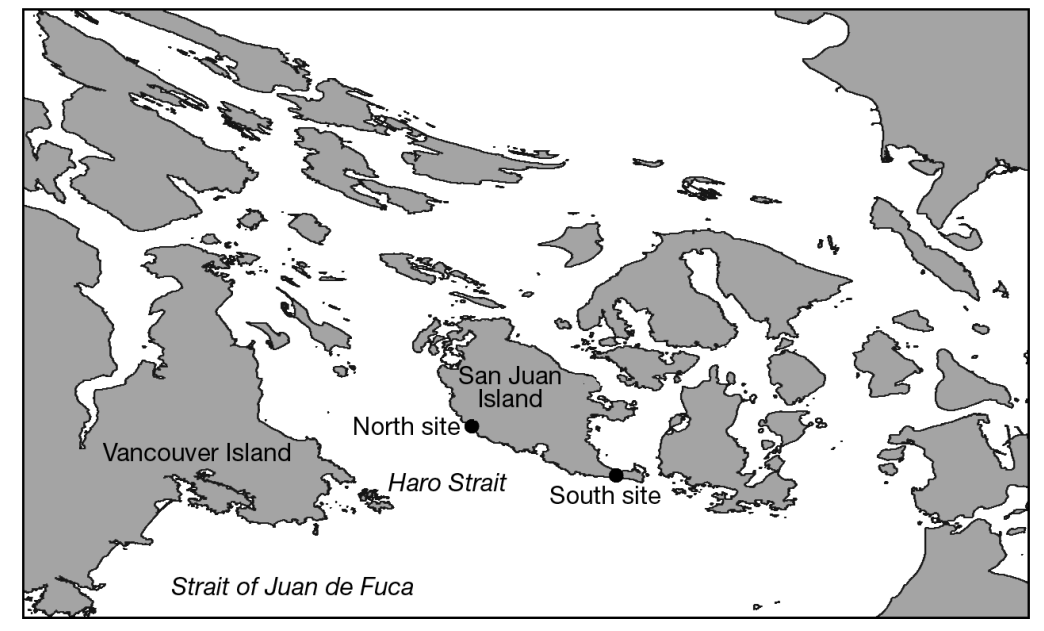

Fig. 1. Study area showing the North and South theodolite sites (•) 
In addition to recording positions of boats and whales, activity states, behavioural events (e.g. respirations and surface active behaviours such as breaches) and other notes about data entry errors or boat and whale activities were recorded (Williams et al. 2002a,b). Boat and whale data were summarised for each track so that each track was represented only once in the analyses.

Statistical analysis. Independent variables included those related to: Time (YEAR, MONTH); Location (SITE); Oceanographic Conditions (tide height, TIDE) and magnitude and direction of current (CURRENT); Focal Animal (AGE, SEX, POD); and Vessel Traffic (Point of Closest Approach [PoCA], Overall Boat Count within the field of view [BOATS]); number of boats within 100, 400 and $1000 \mathrm{~m}$ of the focal whale (SUM100, SUM400 and SUM1000, respectively). These variables are largely self-explanatory, but calculation of vessel-related candidate explanatory variables is described in greater detail in Williams et al. $(2002 a, b)$ and Williams \& Ashe (2007). The terms YEAR and SUM100 entered the model as factors, because relatively few levels of the variables were observed. Other factors in the model included SITE, SEX and POD. All other terms entered the model as continuous covariates.

Dependent (i.e. whale response) variables were calculated using methods described previously (Williams et al. 2002a,b, Williams \& Ashe 2007) and included: (1) Inter-breath interval or dive time, respiration analysis (RESP) - a mean time between breaths was calculated (in s) for each track. Only tracks lasting more than $800 \mathrm{~s}$ were included in the analysis to ensure the data reliably reflected the ongoing breathing pattern (Bain 1986, Kriete 1995); (2) Swimming speed (SPEED): The average swimming speed (reported in $\mathrm{km} \mathrm{h}^{-1}$ ) of the whale was obtained by dividing the total surface distance travelled by the duration of the tracking session.

Two measures of path predictability were calculated: a directness index (DI) and a deviation index (DEV). DI estimates path tortuosity (or its inverse, linearity) on the scale of an entire tracking session, and is generated by dividing the distance between end points of a path (i.e. crow's flight distance) by the cumulative surface distance covered during all dives and multiplying by 100. DI is the ratio of the diameter of a path to its perimeter, and ranges from zero (a circular path) to 100 (a straight line). DEV estimates path tortuosity (or its inverse, smoothness) on the scale of adjacent surfacings. For each surfacing in a track, we calculated the angle between the path taken during a dive and the straight-line path predicted by the dive before it. DEV is the mean of the absolute value of these discrepancies in degrees (potentially ranging from 0 to 180), during the entire track.
Surface active behaviour (SAB) was modelled in 2 ways: the rate at which bouts occurred, and the probability that a bout would occur in a track. The rate of $\mathrm{SAB}$ was determined by recording each time that surface-active events such as spy-hopping, tail-slapping or breaching occurred, and presenting this as an average rate of events expected per hour. The occurrence of SAB (SAB.1.0) was determined by recording each time that surface-active events occurred and presenting this as a binary (yes-no) response variable.

Analysis of theodolite data from focal individuals. We modelled whale behaviour using generalised additive models (GAMs) in the multiple generalised crossvalidation package (mgcv) for program R (Wood 2001, 2006). The GAM framework was chosen because it allowed us to incorporate continuous and categorical explanatory variables, response variables from nonnormal distributions, and because some of the relationships between explanatory and response variables were expected to be non-linear (Williams \& Ashe 2007). The mgcv package allows GAMs to be fitted in $\mathrm{R}$ using regression splines for the smooth terms of each explanatory variable, with each spline carrying a penalty for excessive flexibility (Wood 2001, 2006). All models were fitted using the cubic regression spline basis, which is recommended for cases where the ratio of variables to observations is large or where convergence problems are expected (Wood 2006). Flexibility was determined by the number of 'knots' (approximately one higher than the estimated degrees of freedom, edf) for each model term, between which the functional, or smoothed, relationship was modelled. The amount of flexibility given to any model term was determined by minimising the generalised cross-validation (GCV) score of the whole model (i.e. given the other terms in the model). The GCV score penalises models for being over-parameterised.

The default smoothing value used for splines was the default value set by package mgcv, 10 knots in each spline, corresponding to 9 df (Wood 2001), with an additional penalty (gamma $=1.4$ ) to prevent oversmoothing, which is recommended in cases with relatively few observations per variable (Wood 2006). The shape of the smooth terms in the GAM was solved as a likelihood maximisation problem using penalised iteratively reweighted least squares (Wood 2006). In the case of cubic regression splines, knots are placed evenly throughout the covariate values, with values connected between knots by sections of cubic polynomials.

Histograms of the response variables were used to determine the appropriate family distribution and link function. All response variables were bounded by zero (i.e. subject to the constraint that they could not be negative) and most showed evidence of skew. Given that some were derived variables so that their underly- 
ing distributions were unknown, the models were fitted using quasi frameworks which estimated the dispersion parameter in a maximum likelihood framework (in contrast to the degree of smoothing, which was estimated using penalised iterative least squares). The following summarises our model specification procedure adopted for each of the response variables, $Y$, during this study, using the framework proposed by Wood (2001):

(1) A fully saturated model was fitted to the data: $\mathrm{Y}$ $\mathrm{YEAR}+\mathrm{s}(\mathrm{MONTH})+\mathrm{SITE}+\mathrm{POD}+\mathrm{s}(\mathrm{AGE})+\mathrm{SEX}+$ $\mathrm{s}(\mathrm{TIDE})+\mathrm{s}($ CURRENT $)+\mathrm{s}(\mathrm{PoCA})+\mathrm{s}($ BOATS $)+$ SUM100 + s(SUM400) $+\mathrm{s}($ SUM1000), with the default degree of smoothing (10 knots, $9 \mathrm{df})$.

(2) Model fit was assessed using the summary.gam and plot.gam functions in $\mathrm{mgcv}$, which showed coefficients, GCV score, explanatory power (deviance explained) and fit (residual plots).

(3) For each linear term, we examined the parameter coefficient (slope) to see whether it was near 0 and the significance term to see whether it was near 1 . If so, the term was removed to see whether the GCV score decreased and the explanatory power of the model increased. If both criteria were fulfilled, the term was dropped from the model. If no improvement was detected by removing the term, then it remained in the model.

(4) For each smooth model term, the estimated number of df was examined to see if it was near 1 . We examined the $95 \%$ CIs for that term to see whether they included 0 across the range of observations. If so, the term was dropped temporarily, to see whether the GCV score dropped and the explanatory power of the model increased.

(5) A term was dropped from the final model if it satisfied all 3 of the conditions in Step 4 (i.e. edf $\approx 1 ; 95 \%$ CIs include 0 across range of $x ;$ and dropping the term decreased the GCV score and increased the deviance explained). If the first criterion was met (edf $\approx 1$ ), but not the other 2 , then the smooth term was replaced by a linear term.

These guidelines for including or dropping terms are particularly well suited for cases in which multicollinearity in explanatory variables is suspected (Wood 2001). Correlations between explanatory variables require that the evaluation of whether to drop a model term (Step 5) be conducted one at a time, starting with the variable that has least support from the data for inclusion in the model (i.e. p nearest 1). We did explore the use of generalised additive mixed-effects models (GAMMs, Wood 2006) in which individual identity was treated as a random effect, but in all cases explanatory power (deviance explained) of any given model was approximately an order of magnitude lower than it was for its GAM counterpart. In other words, there was evidence from the data that substantial within-individual variability existed and insufficient support from the data to justify including individual identity as a covariate.

Plots were produced for all selected models (see Figs. 2 to 7 ) using the plot.gam function in mgcv. The $X$-axis for each plot contains a rugplot, in which small ticks mark locations of observations. On the $y$-axis 0 corresponds to no effect of the covariate on the estimated response, values above 0 indicate positive correlation, while values below 0 indicate negative correlation. The $y$-axis is labelled $s$ (covariate name, estimated df) indicating that the curve is smoothed. The dashed lines represent $\pm 2 \mathrm{SE}$, or roughly $95 \% \mathrm{CI}$.

\section{RESULTS}

\section{Theodolite tracking of focal individuals}

We collected 42 tracks in 2003, 77 tracks in 2004, and 67 tracks in 2005 that were of sufficient quality and duration to use in the analysis (Table 1). Roughly $50 \%$ of the individuals in the population were sampled at least once during the 3 seasons. Whales were tracked for an average of 25.2 min over $2.6 \mathrm{~km}$. Only 25 tracks met the criteria for no-boat observations (2 in 2003, 19 in 2004 and 4 in 2005, Table 1).

\section{Results of GAM-based analyses of focal animal behaviour}

RESP. The model that fitted the respiration data best included 2 vessel traffic variables (SUM400 and BOATS) and 1 whale-related variable (AGE) (Table 2, Fig. 2). The model described the variation in mean respiratory interval reasonably well, in that it was able to account for $25.7 \%$ of the deviance. One traffic variable (SUM400) entered the model as a smooth term, while the other (BOATS) entered the model as a linear term.

As the number of boats increased, the inter-breath interval showed a significant tendency to decrease (Table 2). Note that coefficients for linear terms are on the scale of the units of the original variables, so it is not possible to compare the magnitude of the effect of boat number and proximity by comparing the magnitudes of coefficients in Table 2 (because the former is measured in counts, while the latter is measured in $\mathrm{m}$ ).

Dive times tended to be shorter when few boats were present within $400 \mathrm{~m}$ of the focal whale, and increased as number of boats increased (Fig. 2). When $>8$ boats were present within $400 \mathrm{~m}$, mean inter-breath interval declined, but this relationship became non-significant (i.e. CIs comfortably spanned 0 ) with large numbers of boats (8 to 14). 
Table 1. Orcinus orca. Sample size of theodolite tracks

\begin{tabular}{|c|c|c|c|c|}
\hline \multirow[t]{2}{*}{ Parameter } & \multicolumn{4}{|c|}{ Sample size (no. of tracks) } \\
\hline & 2003 & 2004 & 2005 & Total \\
\hline \multicolumn{5}{|l|}{ Study site } \\
\hline North & 28 & 52 & 47 & 127 \\
\hline South & 14 & 25 & 20 & 59 \\
\hline \multicolumn{5}{|l|}{ Month } \\
\hline May & 0 & 33 & 14 & 47 \\
\hline June & 0 & 13 & 27 & 40 \\
\hline July & 4 & 19 & 26 & 49 \\
\hline August & 16 & 12 & 0 & 28 \\
\hline September & 22 & 0 & 0 & 22 \\
\hline \multicolumn{5}{|c|}{ Track duration (min) } \\
\hline $13.3-20$ & 15 & 27 & 16 & 58 \\
\hline$>20$ & 27 & 50 & 51 & 128 \\
\hline \multicolumn{5}{|c|}{ Sex of focal animal } \\
\hline Female & 7 & 39 & 16 & 62 \\
\hline Male & 27 & 38 & 41 & 106 \\
\hline Unknown & 8 & 0 & 10 & 18 \\
\hline \multicolumn{5}{|c|}{ Pod of focal animal } \\
\hline $\mathrm{J}$ & 8 & 44 & 19 & 71 \\
\hline $\mathrm{K}$ & 4 & 9 & 11 & 24 \\
\hline $\mathrm{L}$ & 21 & 24 & 26 & 71 \\
\hline Unknown & 9 & 0 & 11 & 20 \\
\hline \multicolumn{5}{|c|}{ Traffic (no. of unique boats in theodolite track) } \\
\hline 0 & 2 & 19 & 4 & 25 \\
\hline 1 & 2 & 2 & 10 & 14 \\
\hline 2 & 0 & 4 & 2 & 6 \\
\hline 3 & 3 & 6 & 3 & 12 \\
\hline 4 & 1 & 6 & 2 & 9 \\
\hline 5 & 6 & 2 & 2 & 10 \\
\hline $6-10$ & 17 & 15 & 13 & 45 \\
\hline $11-15$ & 6 & 11 & 16 & 33 \\
\hline $16-20$ & 1 & 3 & 4 & 8 \\
\hline $21-25$ & 2 & 2 & 9 & 13 \\
\hline $26-30$ & 2 & 3 & 1 & 6 \\
\hline 31-35 & 0 & 1 & 0 & 1 \\
\hline $36-40$ & 0 & 1 & 1 & 2 \\
\hline $41-45$ & 0 & 2 & 0 & 2 \\
\hline \multicolumn{5}{|c|}{ Minimum no. of focal individuals sampled ${ }^{a}$} \\
\hline & 13 & 34 & 24 & 45 \\
\hline Total & 42 & 77 & 67 & 186 \\
\hline aignoring unl & & & & \\
\hline
\end{tabular}

SPEED. The selected model included only the maximum number of boats within $400 \mathrm{~m}$ of the focal animal (Table 2, Fig. 3). The model was able to account for only $7.3 \%$ of the deviance. The non-linear relationship between swimming speed and MAX400 was fairly flat until the number of boats within $400 \mathrm{~m}$ reached approximately 8 , at which point swimming speed increased (Fig. 3).

DI. The model that fitted the path DI data best included 2 vessel-related variables (PoCA and BOATS), as well as AGE (Table 2, Fig. 4). The model demonstrated weak power to describe variation in DI, amounting to only $5.8 \%$ of the deviance explained. The variables BOATS and PoCA entered the model as
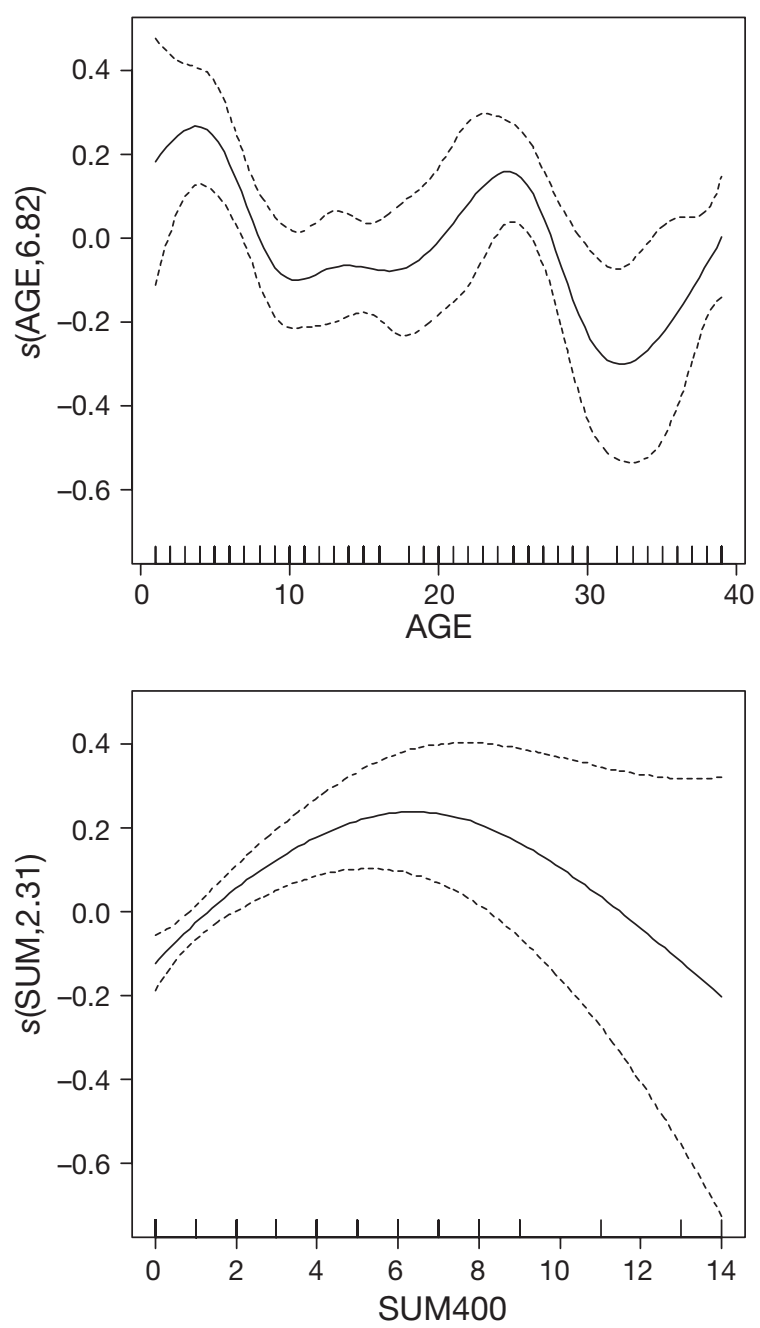

Fig. 2. Relationships between smoothed component (solid line) of the explanatory variables ( $x$-axis) used in the fitted generalised additive model and the response variable, meantime between breaths (RESP). The $x$-axis for each plot contains a rugplot, in which small ticks mark locations of observations. On the $y$-axis, 0 corresponds to no effect of the covariate on the estimated response, values $>0$ indicate positive correlation, while values $<0$ indicate negative correlation. The $y$-axis is labelled $s$ (covariate name, estimated df) indicating that the curve is smoothed. SUM400: number of boats within $400 \mathrm{~m}$ of the focal whale. Dashed lines: $\pm 2 \mathrm{SE}$, or roughly $95 \% \mathrm{CI}$

linear terms with positive slope. Fig. 4 shows the nonlinear relationship between AGE and DI. While the CIs span 0 across the range of AGE, model fit was improved by retaining this term.

DEV. The model that fitted the path DEV data best included 1 boat-related variable (BOATS), 1 whalerelated variable (AGE), and 1 environmental variable (TIDE). Model fit was improved by dropping the intercept term. The model demonstrated weak power to describe variation in DEV, amounting to only $6.6 \%$ of 
Table 2. Orcinus orca. Summary of selected models describing whale behaviour as linear and smooth functions of covariates. Model terms are described in 'Materials and methods.' Each row represents a candidate explanatory variable, and each column represents a response variable. The value in each cell represents the slope of linear terms (numbers) or the degree of smoothing ( $s$ [estimated degrees of freedom]) for smooth terms that were included in the model. Note that coefficients for linear terms are on the scale of the units of the original variables. Significance of each model term: ${ }^{* * *} p=0-0.001 ;{ }^{* *} p=0.001-0.01$; ${ }^{*} \mathrm{p}=0.01-0.05 ; \mathrm{p}=0.05-0.1 ;$ no symbol, $\mathrm{p}>0.1 ;(-)$ term dropped from the model; $\mathrm{N}$ : sample size for the model

\begin{tabular}{|c|c|c|c|c|c|c|}
\hline & RESP & SPEED & DI & $\mathrm{DEV}$ & SAB & SAB.1.0 \\
\hline Intercept & $4.04^{* * *}$ & $1.85^{* * *}$ & $-0.38^{* * *}$ & - & - & - \\
\hline YEAR & - & - & - & - & - & - \\
\hline SITE & - & - & - & - & - & - \\
\hline MONTH & - & - & - & - & $0.181^{* * *}$ & - \\
\hline POD & - & - & - & - & - & - \\
\hline SEX & - & - & - & - & - & - \\
\hline AGE & $s(6.82)^{* * *}$ & - & $s(1.72)$ & $s(1.96)^{*}$ & $-0.027^{*}$ & - \\
\hline TIDE & - & - & - & $-0.001^{*}$ & $s(3.41)^{* *}$ & - \\
\hline CURRENT & - & - & - & - & - & $s(7.64)$ \\
\hline SUM100 & - & - & - & - & - & - \\
\hline SUM400 & $s(2.31)^{* * *}$ & $s(4.75)^{*}$ & - & - & $s(2.66)$ & $s(2.69)$ \\
\hline SUM1000 & - & - & - & - & - & - \\
\hline PoCA & - & - & $3.6 e-5^{*}$ & - & - & $-0.003^{*}$ \\
\hline BOATS & $-0.0132^{* *}$ & - & $0.0068^{*}$ & $-0.01^{*}$ & - & $-0.065^{* *}$ \\
\hline Family & Quasi & Quasi & Quasi & Quasi & Quasipoisson & Quasibinomial \\
\hline Link function & $\log$ & $\log$ & $\log$ & $\log$ & $\log$ & Logit \\
\hline Deviance explained (\%) & 25.7 & 7.30 & 5.80 & 6.60 & 20.20 & 14.30 \\
\hline $\mathrm{N}$ & 153 & 153 & 185 & 186 & 153 & 153 \\
\hline
\end{tabular}

the deviance explained. BOATS entered the model as a linear term with negative slope, indicating that whales exhibited relatively smooth paths when few boats were observed close to the whale and more erratic paths when many boats were present (Table 2). The effect of TIDE on path deviation suggests that there may be something of ecological importance (perhaps foraging activity) reflected in these data and warrants further attention.

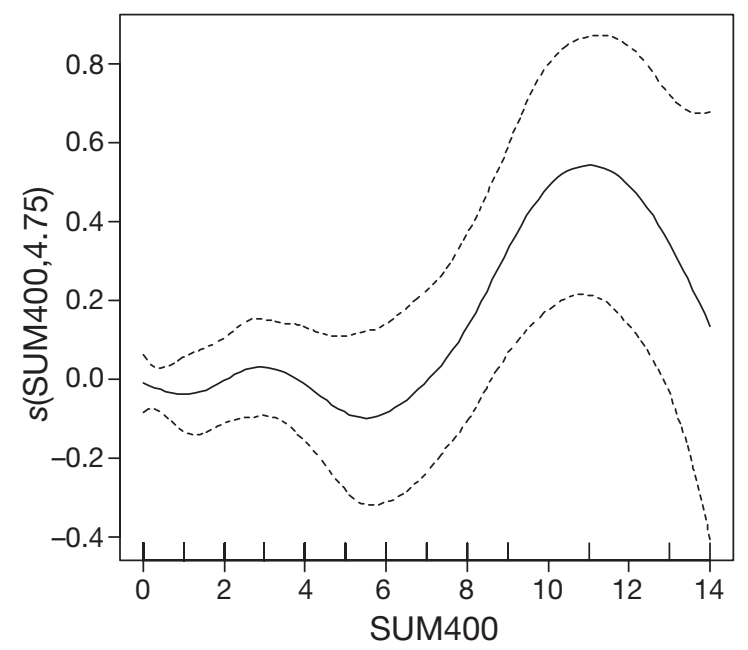

Fig. 3. Relationship between smoothed component (solid line) of the explanatory variable ( $x$-axis) used in the fitted generalised additive model and the response variable, swimming speed (SPEED). SUM400: number of boats within $400 \mathrm{~m}$ of the focal whale. Dashed lines: \pm 2 SE (roughly $95 \%$ CI). For further information see Fig. 2
The smooth term relating DEV to AGE in the selected model is shown in Fig. 5. The spline shows weak evidence that swimming paths showed a nonlinear relationship with $\mathrm{AGE}$; however the CIs span 0 across a wide range of AGE. The term was retained because dropping it increased the GCV score and reduced the model's explanatory power.

Rate of SAB. The results for $\mathrm{SAB}$ are shown in Table 2 and Fig. 6. The model that best fitted the SAB

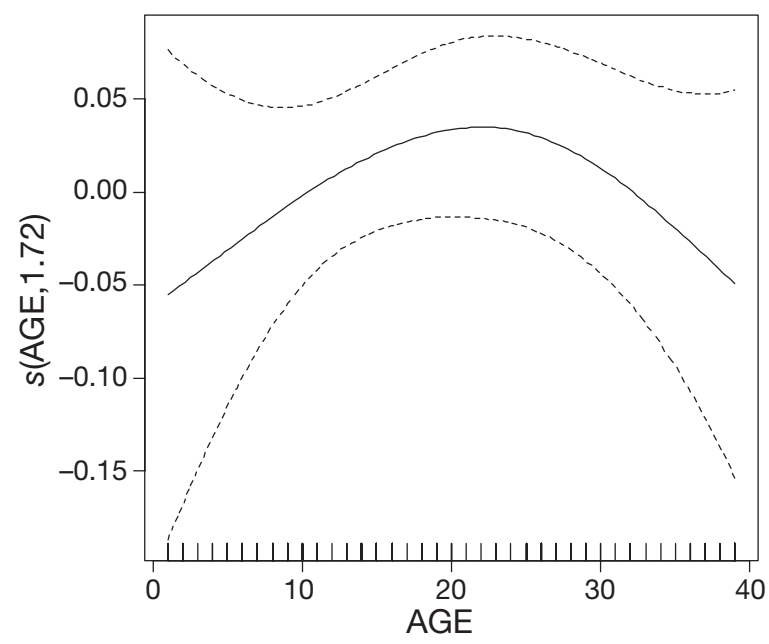

Fig. 4. Relationships between smoothed component (solid line) of the explanatory variable ( $x$-axis) used in the fitted generalised additive model and the response variable, path directness index (DI). AGE: age of the focal whale. Dashed lines: \pm 2 SE (roughly $95 \%$ CI). For further information see Fig. 2 


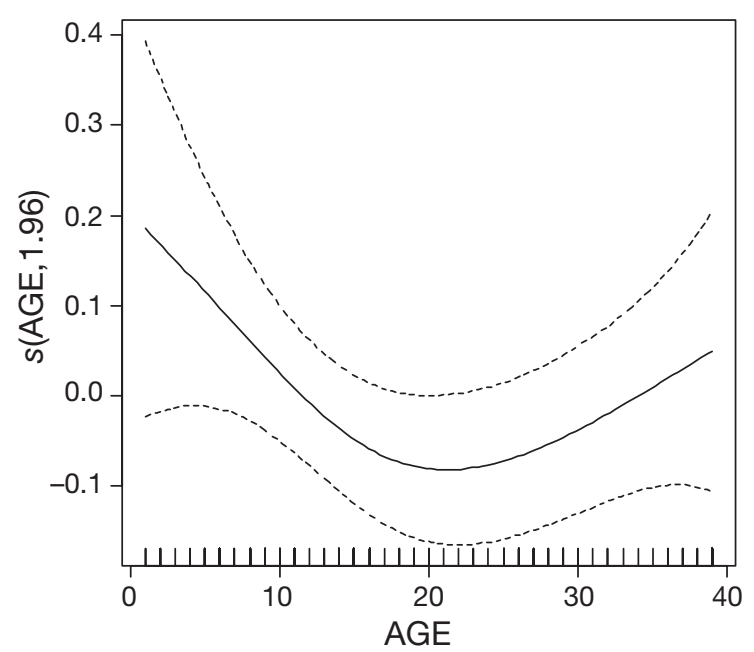

Fig. 5. Relationship between smoothed component (solid line) of the explanatory variable ( $x$-axis) used in the fitted generalised additive model and the response variable, path deviation index (DEV). AGE: age of the focal whale. Dashed lines: \pm 2 SE (roughly $95 \% \mathrm{CI}$ ). For further information see Fig. 2

data included only 1 vessel traffic variable (SUM400), but also TIDE, MONTH and AGE (Table 2, Fig. 6). Model fit was improved by dropping the intercept term. The model demonstrated modest power to describe variation in rates of $\mathrm{SAB}$, amounting to $20.2 \%$ of the deviance explained. Analysis suggests that rates of $\mathrm{SAB}$ declined with AGE, but increased as the season progressed (MONTH). The effect of boats (SUM400, Fig. 6) was non-linear; SAB was highest when boats were absent, low when intermediate numbers of boats approached the whale within $400 \mathrm{~m}$ (SUM400), and higher again with large numbers of boats. Note, how- ever, that the CIs spanned 0 across much of the range (Fig. 6). The non-linear effect of TIDE on rate of SAB suggests that there may be something of ecological importance (perhaps foraging activity) reflected in these data (with very high rates of SAB observed at the highest tides) and warrants further attention.

SAB.1.0. The results for SAB.1.0 are shown in Table 2 and Fig. 6. Model fit was improved by dropping the intercept term. The model demonstrated modest power to describe variation in the probability that a bout of SAB would or would not occur, amounting to $14.3 \%$ of the deviance explained. The analysis of SAB.1.0 suggests surface active events were most likely to occur when the number of boats within $400 \mathrm{~m}$ of the whale was small (SUM400; Fig. 7), although the CIs spanned 0 across much of the range. The probability of bouts of SAB occurring was negatively related to both PoCA and BOATS, indicating that surface active events were less likely to occur as boat number increased, but more likely to occur as boats got closer to the whale.

The non-linear effect of CURRENT on the SAB.1.0 suggests that there may be something of ecological importance (perhaps foraging activity) reflected in these data (with very high probability of SAB occurring observed at the strongest ebb current) and warrants further attention.

Age was not a factor in SAB.1.0, perhaps suggesting that the probability of engaging in $\mathrm{SAB}$ is equal for all age classes, but that younger animals tend to be more active once they get started. Similarly, pods may be equal in their probability of initiating a bout of SAB, but differ in the number of events performed once a bout is initiated.
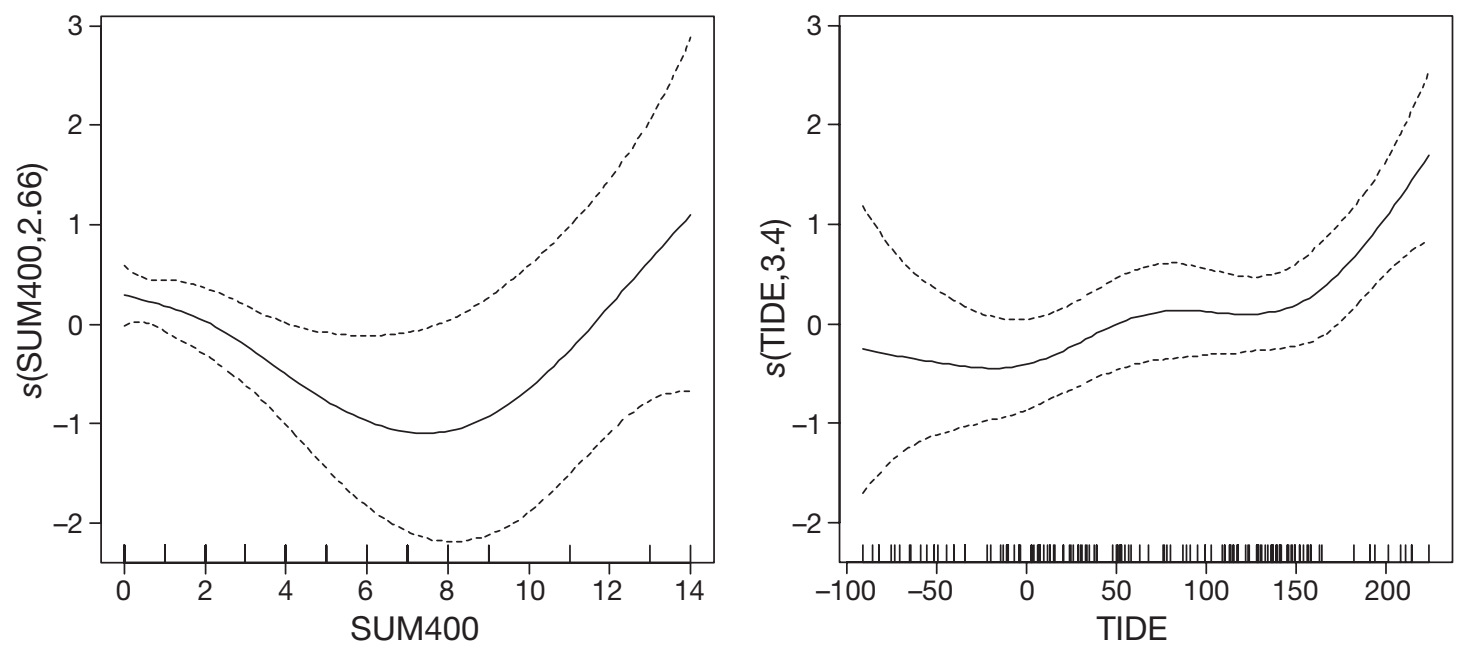

Fig. 6. Relationships between smoothed components (solid line) of the explanatory variables ( $x$-axis) used in the fitted generalised additive model and the response variable, rate of surface active behaviour (SAB). SUM400: number of boats within $400 \mathrm{~m}$ of the focal whale. TIDE: tide height. Dashed lines: \pm 2 SE (roughly $95 \%$ CI). For further information see Fig. 2 

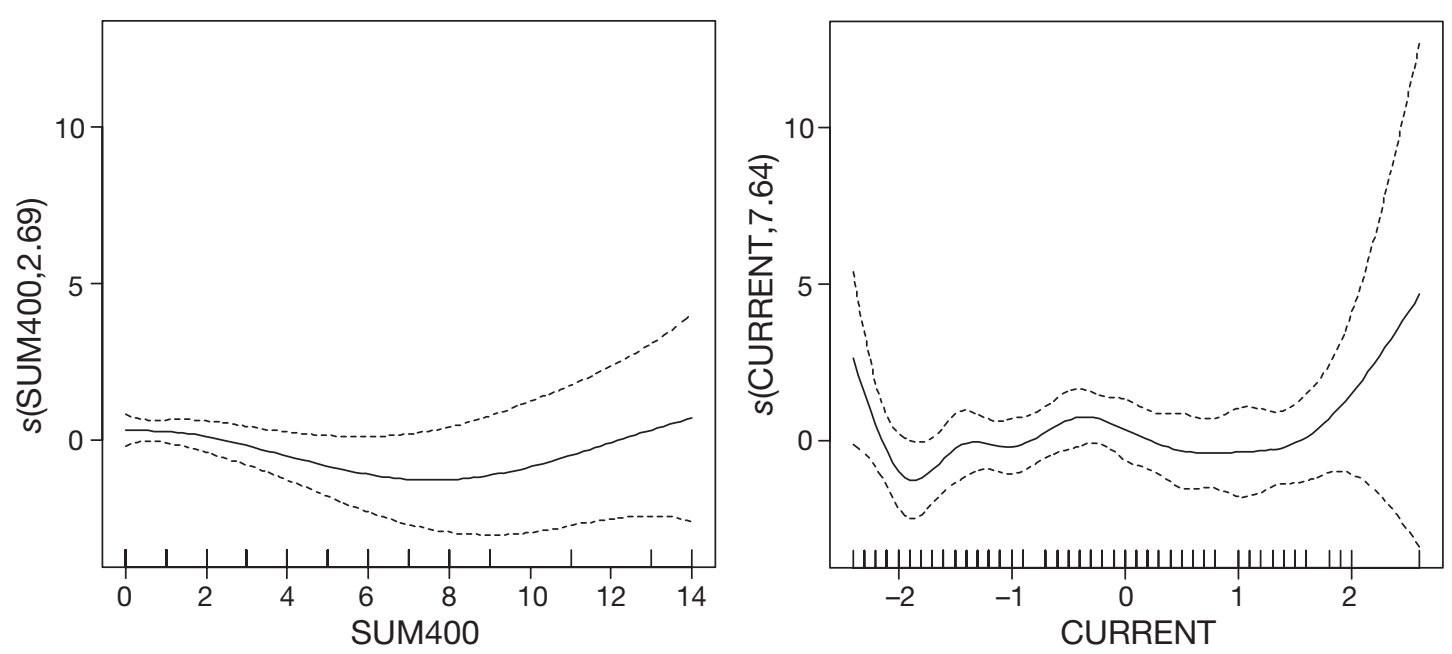

Fig. 7. Relationships between smoothed components (solid line) of the explanatory variables ( $x$-axis) used in the fitted generalised additive model and the response variable, probability of a bout of surface active event occurring (SAB.1.0). SUM400: number of boats within $400 \mathrm{~m}$ of the focal whale. CURRENT: direction of current. Dashed lines: \pm 2 SE (roughly 95\% CI). For further information see Fig. 2

\section{DISCUSSION}

Despite a model specification approach that penalised over-parameterisation, all 6 models fitted the killer whale behaviour data better with boat variables included than when boat variables were excluded. The models lend support to the conclusion that boats exerted a small but significant effect on behaviour of southern resident killer whales in 2003 to 2005, and that the relationships were complex and often non-linear.

Williams et al. (2002b) and Williams \& Ashe (2007) suggested that vessel number and vessel proximity were different dimensions of vessel traffic, and that a killer whale's response to changes in vessel number is likely to occur independently of its response to changes in proximity, and vice versa. As such, an increase in proximity need not have the same effect as an increase in number. This study supports that suggestion. In all 6 cases, SUM100 and SUM1000 were dropped from the models, while SUM400 was retained in 4 of 6 cases. Similarly, PoCA was retained in only 2 of 6 models, but in both cases in which the term was retained, it offered little explanatory power (Table 2).

We also observed several non-linear effects of boat traffic on whale behaviour. Qualitatively, it appeared there was a baseline distribution of behaviour when boats were absent, a trend with intermediate numbers of boats (from 1 to about 3 vessels), and the opposite trend when the number of vessels was large $(>10)$.

The complexities described above may account for inconsistencies among studies, many of which simply compared a vessel-present to a vessel-absent condition. Williams \& Ashe (2007) demonstrated experimen- tally that the response of northern residents to approach by few (1 to 3 ) boats was opposite to that of many (4 to 17) boats, and that the average of these opposing responses would be statistically indistinguishable from no response. In southern residents, our results suggest that the number of boats within $400 \mathrm{~m}$ of the focal whale helped explain much of the variability in whale behaviour (SUM400 appeared in 4 of our 6 models), but current guidelines for this population focus more on proximity than crowding (although proximity appeared in only 2 of our 6 models).

The relationships between path directness and vessel traffic have appeared consistently in studies such as the present one (Williams et al. 2002a,b, Williams \& Ashe 2007). These patterns are consistent with whales making concerted efforts to evade boats: swimming paths became less direct as boats approached close to the whales, but more direct as number of boats increased (Table 2). Our analyses confirmed that both vessel number and proximity were significant factors, even after taking natural factors into account. Altering swimming paths may lead to an increase in energy expenditure if the decreased DI results in an overall increase in time spent travelling over time spent resting (Williams et al. 2006).

The path DEV decreased as the number of boats increased (Table 2). This is consistent with previous multiple-vessel experiments that demonstrated that avoidance responses which are effective with few boats may not be effective with many boats (Williams \& Ashe 2007). The DEV would be expected to be relatively high during socialising and foraging, and TIDE was a natural factor correlated with DEV. Felleman et 
al. (1991) suggested that foraging strategies of whales should take into account current-related movements of their salmonid prey. This relationship merits additional investigation.

Reported changes in breathing patterns as a function of boats have been inconsistent. The GAM analysis suggests that inter-breath interval increases with increasing vessel number when the number of vessels is small (from 1 to about 6 vessels), but decreases when the number of vessels is large (up to 12 vessels). This U-shaped response pattern may account for the inconsistent results reported in various studies. There may be alternative tactics employed that vary depending on vessel number and proximity (Williams \& Ashe 2007). Vessel proximity did not enter directly as a factor, although boat counts at different distances entered separately, suggesting that proximity has some relevance. It is possible that this index is more sensitive to distances throughout the track relative to brief close approaches than other indices are, or that respiration rate (as a physiological variable) may be more strongly related to swimming speed and activity level than boat traffic. Additional data will be needed to confirm whether the result reported here is robust in a wider range of conditions.

Changes in SAB have been significant in many studies, although the direction of the change varies from one study to another. Our results suggest the inconsistency may be due to differences in methodology. For example, our work and that of Williams et al. (2002b) suggest that $\mathrm{SAB}$ is maximised when 1 or a small number of boats approach closely, but SAB may be inhibited by other configurations of vessels. Data collected when boats are primarily in an inhibitory configuration may find that vessels reduce rates of SAB. Alternatively, studies that pool all configurations may find no effect (Williams \& Ashe 2007).

The analysis is further complicated by the relationship between track duration and measured values. Analysis of rates may need to be limited to longer tracks than some of those used here. Longer tracks would also be helpful for one-zero sampling, as that would allow the subdivision of tracks into multiple short segments. The tendency of SAB to occur in bouts, along with the fact that $\mathrm{SAB}$ is a somewhat artificial class composed of behaviour patterns (breaching, spyhopping, tail-slapping, etc.) with a wide-range of functions, make it difficult to address these behaviour patterns with statistical rigour. Nonetheless, the increased probability of $\mathrm{SAB}$ occurring in the presence of vessels appears robust, as the effect is large and present in numerous datasets.

One could speculate that threat displays consisting of SABs such as breaches, slaps, and fluke lifts (Tavolga 1966, Norris et al. 1994, Lusseau 2006a,b) in- creased when vessels were close but not close enough to trigger an escape response. At greater distances, $\mathrm{SAB}$ could be reduced to avoid attracting the attention of vessel operators. Baseline rates would reflect the use of $\mathrm{SAB}$ for purposes independent of vessels such as communication, foraging, and non-communicative behaviour. Some SABs, such as breaching, require increased energy expenditure, so this variable should be considered when calculating cumulative effects.

The trend in swimming speed with respect to vessel traffic has been inconsistent across studies (e.g. contrast Kruse 1991 with Williams et al. 2002b). The GAM analysis suggested that the number of boats within $400 \mathrm{~m}$ could be important. Given the potential for changes in swimming speed to carry energetic costs to whales, as well as reflecting their physical condition, the factors influencing swimming speed deserve more careful assessment.

Felleman et al. (1991) found that resident killer whales were approximately equally likely to be oriented with, against or non-oriented with respect to tidal currents. However, killer whales were found to change their direction of travel most often near slack tide; they were 7 times more likely to change direction within an hour of slack tide than one would expect from chance (Felleman et al. 1991). Although TIDE and CURRENT were dropped from our models of RESP, SPEED and DI, these oceanographic variables were included in models describing variability in DEV and $\mathrm{SAB}$. One interpretation of this finding is that SABs vary inconsistently with boat traffic, but play an important role in foraging ecology of resident killer whales.

The results reported here exhibit similarities and differences with other species. Variation in behavioural responses with boat proximity appears to be relatively common (Nowacek et al. 2001). Increases in travel and $\mathrm{SAB}$ are also commonly found. In contrast, measures of swimming speed have varied among species and among studies within species, with some studies reporting increases (Orcinus orca: Kruse 1991; Tursiops truncatus: Nowacek et al. 2001), some reporting no change $(O$. orca: present study; Globicephala macrorhynchus, Stenella coeruleoalba, Steno bredanensis: Ritter 2003), and some reporting both increases and decreases depending on vessel speed (Stenella frontalis, T. truncatus: Ritter 2003). Some species show increased dive times as reported here (e.g. Eschrichtius sp.: Sumich 1983), while other species shorten dives in the presence of boats. Some species are displaced from regions by vessels (e.g. Tursiops spp.: Allen \& Read 2000, Bejder et al. 2006), in contrast to resident killer whales, which continued to use the same range in the presence of vessels.

The present study found evidence of small but statistically significant changes in behaviour in the pres- 
ence of vessels. At first glance, these findings raise philosophical questions about the use of advanced statistics in detecting disturbance. If the detection of vessel effects requires such delicate methodology, are these effects really worth mitigating against? In practice, scientists and managers would be more comfortable if management decisions were based on the results of experiments conducted on northern residents. However, this system is now so heavily saturated by boats (Table 1) that experiments are all but impossible, and relatively sophisticated modelling frameworks were required to cope with confounding effects and estimate partial contribution of boat traffic to describing whale behaviour. The fact that models fitted the data better with boat variables than without, despite penalties for over-parameterisation, indicates that such anthropogenic impacts are real. It remains a management decision to evaluate whether effect sizes are large enough to warrant regulations for vessel operation near killer whales. Given the small population size, the endangered status of the population, and the suggestion that boat traffic can disrupt feeding activities of resident killer whales (Williams et al. 2006, Lusseau et al. 2009), it seems reasonable to us to err on the side of caution when managing boat traffic around southern resident killer whales. Given suggestions that this population may be food-limited (Fisheries and Oceans Canada 2008), minimising boat traffic around whales may also improve foraging efficiency by reducing masking effects of boat noise on echolocation (Bain \& Dahlheim 1994). Clearly, managing boat traffic around whales does not address prey limitation or toxic contamination, but unlike these potentially larger problems, boat traffic is a demonstrated threat that lends itself to immediate mitigation.

Acknowledgments. We thank B. Gleason of San Juan Island National Historic Park for permission to use the South Site (Permits SAJH-2004-SCI-0003 and SAJH-2005-SCI-0003), and the Reiss family for permission to use the North Site. We thank E. Ashe, A. Coleman, B. Cuddihy, A. Foote, M. Foss, J. Hogan, A. Miller, F. Robertson, D. Ross, and R. Taylor for assistance with data collection, and S. Heaslip for assistance with analysis. The Whale Museum's Soundwatch Program, Marine Mammal Monitoring Program (M3) and The Whale Watch Operators Association Northwest assisted with increasing the quantity of no-boat data at our North Site. We give special thanks to D. Noren for her help with the design of this project and review of previous versions of the manuscript. We thank J. Matthiopolous and 3 anonymous reviewers for comments on a previous draft. The data used in this report were funded under National Marine Fisheries Service Contracts No. AB133F03SE0959, AB133F04CN0040 and AB133F05SE3965. The views expressed in this manuscript are those of the authors, and not necessarily those of the funding agency.

\section{LITERATURE CITED}

Allen MC, Read AJ (2000) Habitat selection of foraging bottlenose dolphins in relation to boat density near Clearwater, Florida. Mar Mamm Sci 16:815-824

Bain DE (1986) Acoustic behavior of Orcinus: sequences, periodicity, behavioral correlates and an automated technique for call classification. In: Kirkevold BC, Lockard JS (eds) Behavioral biology of killer whales. Alan R. Liss, New York, p 335-371

Bain DE, Dahlheim ME (1994) Effects of masking noise on detection thresholds of killer whales. In: Loughlin TR (ed) Marine mammals and the Exxon Valdez. Academic Press, San Diego, CA, p 243-256

Bejder L, Samuels A, Whitehead H, Gales N and others (2006) Decline in relative abundance of bottlenose dolphins exposed to long-term disturbance. Conserv Biol 20:1791-1798

> DeNardo C, Dougherty M, Hastie GD, Leaper R, Wilson B, Thompson PM (2001) A new technique for investigating variability in spatial relationships within groups of free ranging cetaceans. J Appl Ecol 38:888-895

Felleman FL, Heimlich-Boran JR, Osborne RW (1991) Feeding ecology of the killer whale (Orcinus orca). In: Pryor KW, Norris KS (eds) Dolphin societies: discoveries and puzzles. University of California Press, Berkeley, CA, p 113-147

Fisheries and Oceans Canada (2008) Recovery strategy for the northern and southern resident killer whales (Orcinus orca) in Canada. Species at Risk Act Recovery Strategy Series, Fisheries and Oceans Canada, Ottawa

Krahn MM, Ford MJ, Perrin WF, Wade PR and others (2004) 2004 Status review of southern resident killer whales (Orcinus orca) under the Endangered Species Act. US Dept Commer, NOAA Tech Memo NMFSNWFSC-62

Kriete B (1995) Bioenergetics in the killer whale, Orcinus orca. $\mathrm{PhD}$ thesis, University of British Columbia, Vancouver

Kruse S (1991) The interactions between killer whales and boats in Johnstone Strait, B.C. In: Pryor, K, Norris, KS (eds) Dolphin societies: discoveries and puzzles, University of California Press, Berkeley, CA p 149-159

Lusseau D (2006a) Why do dolphins jump? Interpreting the behavioral repertoire of bottlenose dolphins in Doubtful Sound, New Zealand. Behav Process 73:257-265

Lusseau D (2006b) The short-term behavioral reactions of bottlenose dolphins to interactions with boats in Doubtful Sound, New Zealand. Mar Mamm Sci 22:802-818

> Lusseau D, Bain DE, Williams R, Smith JC (2009) Vessel trafffic disrupts the foraging behavior of southern resident killer whales Orcinus orca. Endang Species Res. 6:211-221

Norris KS, Würsig B, Wells RS (1994) Social behavior. In: Norris KS, Würsig B, Wells RS, Würsig M (eds) The Hawaiian spinner dolphin. University of California Press, Berkeley, CA, p 103-121

Nowacek SM, Wells RS, Solow AR (2001) Short-term effects of boat traffic on bottlenose dolphins, Tursiops truncatus, in Sarasota Bay, Florida. Mar Mamm Sci 17:673-688

Ritter F (2003) Interactions of cetaceans with whale watching boats - implications for the management of whale watching tourism. M.E.E.R. e.V., Berlin

Sumich JL (1983) Swimming velocities, breathing patterns, and estimated costs of locomotion in migrating gray whales, Eschrichtius robustus. Can J Zool 61:647-652

Tavolga MC (1966) Behavior of the bottlenose dolphin (Tursiops truncatus): social interactions in a captive colony. In Norris KS (ed) Whales, dolphins and porpoises. University of California Press, Berkeley, CA, p 718-730

US Fish and Wildlife Service (2005) Endangered and threatened wildlife and plants: endangered status for south- 
ern resident killer whales. Fed Regist 70:69903-69912 van Ginneken A, Ellifrit D, Balcomb KC (2002) Official orca survey field guide. Center for Whale Research, Friday Harbor, WA

Wiles GJ (2004) Washington State status report for the killer whale. Washington Department Fish and Wildlife, Olympia, WA

Williams R, Ashe E (2007) Killer whale evasive tactics vary with number of boats. J Zool (Lond) 272:390-397

Williams R, Bain DE, Ford JKB, Trites AW (2002a) Behavioural responses of male killer whales to a 'leapfrogging' vessel. J Cet Res Manag 4:305-310

Editorial responsibility: Jason Matthiopoulos, St. Andrews, UK
Williams R, Trites A, Bain DE (2002b) Behavioural responses of killer whales (Orcinus orca) to whale-watching boats: opportunistic observations and experimental approaches. J Zool (Lond) 256:255-270

Williams R, Lusseau D, Hammond PS (2006) Estimating relative energetic costs of human disturbance to killer whales (Orcinus orca). Biol Conserv 133:301-311

Wood SN (2001) mgcv: GAMs and Generalized Ridge Regression for R. R News 1:20-25

Wood SN (2006) Generalized additive models: An introduction with R. Taylor \& Francis, London

Submitted: July 19, 2007; Accepted: September 24, 2008

Proofs received from author(s): December 1, 2008 